CASE REPORTS

\title{
"Osler's phenomenon": misdiagnosing Cushing's syndrome
}

\author{
P De, L M Evans, M F Scanlon, J S Davies
}

Postgrad Med J 2003;79:594-596

The clinical manifestations of Cushing's syndrome can be quite variable and are frequently mistaken, with consequent delayed diagnosis and significant morbidity and mortality. Harvey Cushing described the typical signs and symptoms of Cushing's syndrome but unfortunately attributed the features to myxoedema. The first typical description of a patient with Cushing's syndrome was probably made by Sir William Osler in 1898. Thus delay or misdiagnosis with consequent high morbidity and mortality exemplifies the history of Cushing's syndrome.

Four cases of Cushing's syndrome are described that were associated with deteriorating morbidity because of the considerable delay from first presentation to a secondary care physician to eventual diagnosis. The clinical diagnosis was delayed in all the four patients, although they had symptoms and signs that were missed by a number of primary and secondary care physicians. Trans-sphenoidal surgery resulted in biochemical cure as well as improvement in the accompanying co-morbidity.

Although still rare, the prevalence of Cushing's syndrome is increasing. Increasing clinical awareness and the use of appropriate screening tests should facilitate earlier diagnosis with reduced morbidity and mortality. Although the syndrome is named after Harvey Cushing, Sir William Osler was probably the first to describe it. Therefore, in deference to Osler's contribution to Cushing's syndrome and the work of Harvey Cushing, it is suggested that to the list of the other eponymous conditions of Osler-Weber-Rendu and Osler's nodes, should be added the delay or misdiagnosis of Cushing's syndrome-"Osler's phenomenon".

$\mathrm{H}$ arvey Cushing first described the symptom complex of obesity, diabetes, hirsutism, and adrenal hyperplasia in 1912. ${ }^{1}$ He gave a vivid description of his first patient who was 23 years old and presented with the "most extraordinary appearance". But it was not until 20 years later that he correctly attributed these features to basophilic pituitary adenomata. ${ }^{2}$ Consequently, we now diagnose the typical features of hypercortisolism as Cushing's syndrome, yet Cushing was probably not the first to describe the condition. Turney described a typical case with photographic evidence in 1912, ${ }^{3}$ but the first typical description of a patient with Cushing's syndrome was probably made by Sir William Osler in 1898. He described the typical signs and symptoms of Cushing's syndrome but unfortunately attributed the features to myxoedema. ${ }^{4}$ Thus delay or misdiagnosis with consequent high morbidity and mortality exemplifies the history of Cushing's syndrome. We describe four cases of Cushing's syndrome associated with deteriorating morbidity due to the considerable delay from first presentation to a secondary care physician to eventual diagnosis.

\section{CASE REPORTS \\ Case 1}

A 53 year old woman with a one year history of progressive thinning of scalp hair and weight gain was referred by her general practitioner to a dermatologist who diagnosed male pattern hair loss. Over the next two years both hirsutism and weight gain increased.

Believing she might have Cushing's syndrome after reading an article in the lay press, she prompted a referral to an endocrinologist. On examination, she was plethoric, overweight with an early interscapular pad of fat, had mild facial hirsutism, and a blood pressure of 160/90 mm $\mathrm{Hg}$. Hypercortisolism was confirmed with a high urinary free cortisol concentration of $740 \mathrm{nmol} /$ day (normal $<250 \mathrm{nmol} /$ day) and failure of appropriate suppression of cortisol concentrations after low dose (cortisol $54 \mathrm{nmol} / \mathrm{l}$ ) and high dose (cortisol $52 \mathrm{nmol} / \mathrm{l}$ ) dexamethasone. Magnetic resonance imaging (MRI) of the pituitary appeared normal but Cushing's disease was confirmed with high central to peripheral adrenocorticotrophic hormone (ACTH) concentrations after inferior petrosal sinus sampling.

She underwent trans-sphenoidal hypophysectomy and cure of hypercortisolism was suggested by a postoperative cortisol concentration of $62 \mathrm{nmol} / \mathrm{l}$. After surgery she has made good progress with improvement of her hirsutism and weight loss. She remains on prednisolone $6 \mathrm{mg}$ daily.

\section{Case 2}

A 47 year old woman was admitted as an emergency with poorly controlled diabetes mellitus. The patient reported a one year history of change in her appearance with weight gain, particularly central adiposity and weakness. She was diagnosed with diabetes and hypertension six months before hospitalisation and had been under the care of a diabetologist. She was a non-smoker, drank little alcohol, and her only medical history was that of gestational hypertension.

On examination she had cushingoid features with a proximal myopathy and had a blood pressure of 220/ $120 \mathrm{~mm} \mathrm{Hg}$. Investigations confirmed hypercortisolism with loss of diurnal variation of cortisol and failure of cortisol suppression with both low dose $(799 \mathrm{nmol} / \mathrm{l})$ and high dose dexamethasone $(742 \mathrm{nmol} / \mathrm{l})$. MRI of the pituitary was normal. Pituitary dependent Cushing's disease was confirmed by inferior petrosal sinus sampling. She underwent pituitary trans-sphenoidal surgery and cure of hypercortisolism was suggested by a postoperative cortisol concentration of $28 \mathrm{nmol} / \mathrm{l}$. She remains hypopituitary on full replacement. She has made good progress with resolution of her diabetes mellitus and hypertension and has lost $30 \mathrm{~kg}$ in weight.

Abbreviations: $\mathrm{ACTH}$, adrenocorticotrophic hormone; $\mathrm{HbAlC}$, glycated haemoglobin; MRI, magnetic resonance imaging 


\section{Case 3}

A 58 year old woman was referred with a 10 year history of diabetes mellitus, weight gain of over $63.5 \mathrm{~kg}$ and concern related to easy bruising of her skin, arthralgia, and a change in her facial appearance. She had seen multiple specialists over this period of time including a general surgeon (cholecystectomy), an orthopaedic surgeon (joint pains and osteoporosis), a urologist (urinary tract infection), two gastroenterologists (diarrhoea and rapid gastric emptying), three diabetologists, a cardiologist (hypertension and heart failure), a haematologist (iron deficiency anaemia), and two respiratory physicians (shortness of breath).

On examination, she looked typically cushingoid with central obesity, interscapular fat pad, conjunctival oedema, and easy bruising. She was hypertensive and had a profound proximal myopathy. Her glycated haemoglobin (HbAlC) was $12.1 \%$. Investigations confirmed hypercortisolism with raised urinary free cortisol concentrations of $438 \mathrm{nmol} /$ day and a failure of cortisol suppression after both low and high dose dexamethasone. MRI of the pituitary suggested a pituitary microadenoma, and pituitary dependent Cushing's syndrome was confirmed after raised central to peripheral ACTH after inferior petrosal sinus sampling.

After trans-sphenoidal pituitary surgery, the postoperative cortisol concentration was $81 \mathrm{nmol} / \mathrm{l}$ which suppressed to $38 \mathrm{nmol} / \mathrm{l}$ after a $1 \mathrm{mg}$ overnight dexamethasone suppression test. Since surgery she has lost over $20 \mathrm{~kg}$ in weight and has stopped insulin. Her HbAlC is 7\% on $1.5 \mathrm{~g}$ of metformin daily and she continues on DDAVP, prednisolone ( $5 \mathrm{mg}$ daily), and thyroxine.

\section{Case 4}

A 59 year old woman with a five year history of troublesome facial hirsutism was referred to a dermatologist who diagnosed idiopathic hirsutism. She also had a two year history of ischaemic heart disease, hypertension, and mild aortic stenosis for which she had been seeing a cardiologist, a five year history of back pain for which she was seeing an orthopaedic surgeon, and a seven year history of depression for which she had seen a psychiatrist. She was a non-smoker and occasionally drank alcohol. Once again, believing she had Cushing's syndrome after reading an article in lay press, she prompted a referral to the endocrine unit.

On examination, she had truncal obesity, thin skin, facial and abdominal hirsutism, and was hypertensive with a blood pressure of 150/100 $\mathrm{mm} \mathrm{Hg}$.

Endocrine investigations revealed hypercortisolism with non-suppression of cortisol after $1 \mathrm{mg}$ overnight dexamethasone $(101 \mathrm{nmol} / \mathrm{l})$. Hypercortisolism was confirmed with a non-suppressed cortisol concentration after low dose dexamethasone (cortisol $97 \mathrm{nmol} / \mathrm{l}$ ) but adequate suppression after high dose dexamethasone suppression test (cortisol $29 \mathrm{nmol} / \mathrm{l})$. ACTH dependent Cushing's syndrome was suggested by raised 9 am ACTH concentrations (126 ng/l). A pituitary source was confirmed after inferior petrosal sinus sampling which revealed a high central to peripheral ACTH gradient with lateralisation to the right side. MRI merely showed a bulky pituitary gland.

At the time of diagnosis she developed a deterioration of her angina, which required coronary angiography and angioplasty. She underwent successful trans-sphenoidal pituitary surgery with cure of her Cushing's disease as suggested by a postoperative serum cortisol of $27 \mathrm{nmol} / \mathrm{l}$. She remains on prednisolone, thyroxine, atorvastatin, aspirin, growth hormone, and venlafaxine. Her blood pressure is now well controlled and although her facial hirsutism has improved, it still remains a subjective problem.

\section{DISCUSSION}

These four cases illustrate the significant co-morbidity associated with delayed diagnosis of Cushing's syndrome. The delay from the first presentation to the secondary care physician with symptoms that suggested Cushing's syndrome ranged from six months to approximately 10 years. Over this period, all patients experienced a clinical deterioration with increasing weight gain, myopathy, hypertension, hirsutism, and glycaemic control.

Potential explanations for delay or misdiagnosing Cushing's syndrome may relate to the perceived rarity of the condition. Cushing's syndrome has a reported incidence of one in 100000 of the population ${ }^{5}$ and this may be a reasonable estimate of overt Cushing's syndrome. Yet recently, we have witnessed an increasing annual prevalence of Cushing's syndrome at University Hospital Wales, which others have also seen (fig 1). This trend may either reflect a true increasing incidence of Cushing's syndrome, or as seems more likely, increasing awareness with greater diagnostic accuracy. In support of the latter, Leibowitz et al assessed the incidence of Cushing's syndrome among a population of obese patients with diabetes mellitus. ${ }^{6}$ Surprisingly, they demonstrated Cushing's syndrome in three of these subjects, giving a prevalence of $3.3 \%{ }^{6}$ Data from our centre suggest that approximately $2 \%$ of the South Glamorgan population have diabetes mellitus, ${ }^{7}$ and that roughly $50 \%$ of these are obese. Therefore extrapolating Leibowitz's data, we could estimate the incidence of Cushing's syndrome to be as high as 33 per 100000 purely within the diabetic population. However, this too may be an underestimate. Altogether $1 \%$ of the population may have incidental adrenal adenomata on computed tomography, of whom as many as $10 \%$ may have autonomous cortisol secretion, ${ }^{8}$ providing an incidence of Cushing's syndrome or "preclinical" Cushing's syndrome as high as 100 per 100000 population. Therefore, these estimated incidences suggest that Cushing's syndrome may be far commoner than the traditionally accepted figure of one per 100000 . Thus, although increasing awareness may explain the increase in the number of cases of Cushing's syndrome, as the above calculations and our cases illustrate, we may still be missing a considerable number, with the consequent delay in diagnosis associated with increasing morbidity.

Although the clinical description of our four cases would strongly suggest to the reader the diagnosis of Cushing's syndrome, nevertheless, the diagnosis was not considered by numerous primary and secondary clinicians. Typical features

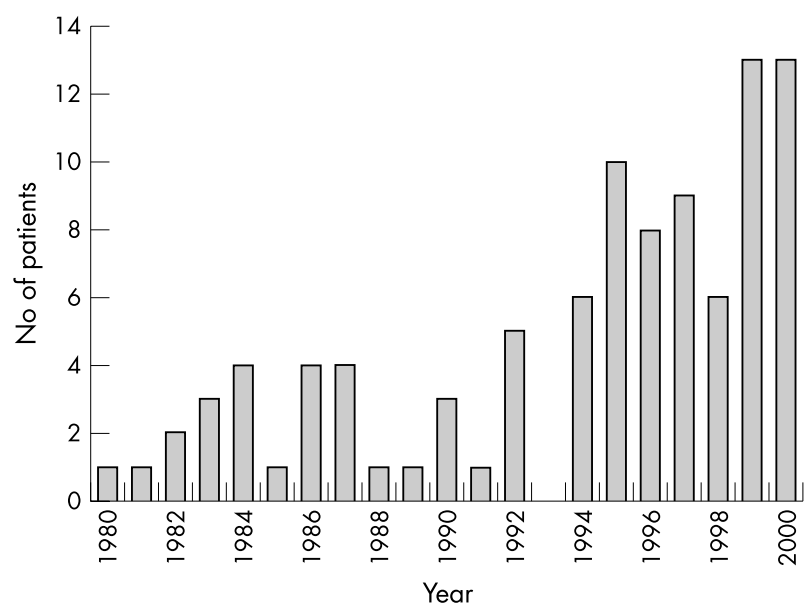

Figure 1 Annual number of patients diagnosed with Cushing's syndrome at University Hospital Wales, Cardiff from 1980 to 2000. 
of Cushing's syndrome include hirsutism, obesity, diabetes mellitus, and hypertension but it appears that there is a reluctance to consider a "rare" diagnosis like Cushing's syndrome with a tendency to attribute such features to pseudo-Cushing's. However, clinical features may be misleading and in certain cases entirely absent (subclinical Cushing's syndrome). Thus, these cases and the suggested increasing prevalence should serve to increase awareness to the potential diagnosis of Cushing's syndrome. A high index of suspicion is necessary and screening tests need to be undertaken on the slightest clinical suspicion. A 24 hour urine free cortisol estimation and the $\mathrm{lmg}$ overnight dexamethasone suppression test are inexpensive screening tests and can be readily performed on an outpatient basis. Both tests have sensitivity and specificity of approximately $95 \%$ for the evaluation of Cushing's syndrome ${ }^{9}{ }^{10}$ and in our series both tests suggested the correct diagnosis.

It is not surprising that Cushing's syndrome is frequently misdiagnosed when one refers to the history of the condition. Although Cushing described his first case in 1912 it was not until 20 years later that he was able to link the typical features with hypercortisolism. Furthermore, Cushing's mentor, Sir William Osler may well have been the first to describe Cushing's syndrome yet misdiagnosed this case as myxoedema. ${ }^{4}$ What is more surprising is that Harvey Cushing may well have attended the lecture at which Osler presented this case (Osler and Cushing were very close, and in fact, Cushing was Osler's biographer). ${ }^{4}$ Therefore, in deference to Osler's contribution to Cushing's syndrome and the work of Harvey Cushing, we would suggest that to the list of the other eponymous conditions, Osler-Weber-Rendu and Osler's nodes, should be added the delay or misdiagnosis of Cushing's syndrome-“"Osler's phenomenon".

\section{Authors' affiliations}

P De, L M Evans, M F Scanlon, J S Davies, Department of Endocrinology, University of Wales College of Medicine, Cardiff, UK

Correspondence to: Dr Pranab De, Histopathology Laboratory, Kuwait Cancer Control Center, Post Box No 42262, 70653 Shuwaikh, Kuwait; deyparanab@hotmail.com,

Submitted 28 October 2002

Accepted 21 January 2003

\section{REFERENCES}

1 Cushing $\mathbf{H}$. The pituitary body and its disorder: clinical states produced by the disorders of the hypophysis cerebri. Philadelphia: JB Lippincott, 1912.

2 Cushing $\mathbf{H}$. The basophil adenoma of the pituitary body and their clinical manifestations (pituitary basophilism). Bulletin of the John's Hopkins Hospital 1932;50:137-95

3 Turney HG. Discussion on disease of the pituitary body. Proc $R$ Soc Med 1913:6: 1xix-1xxvii.

4 Altschule MD. Occasional notes. A near miss-Osler's early description of Cushing's syndrome with, regrettably, no post-mortem examination. N Engl J Med 1980;302:1153-5.

5 Reincke M. Subclinical Cushing's syndrome. Endocrinol Metab Clin North Am 2000;29:43-56.

6 Leibowitz G, Tsur A, Chayen SD, et al. Pre-clinical Cushing's syndrome: an unexpected frequent cause of poor glycemic control in obese diabetic patients. Clin Endocrinolo (Oxf) 1996;44:712-22.

7 Morgan CL, Currie CJ, Stott NC, et al. Estimating the prevalence of diagnosed diabetes in a health district of Wales: the importance of using primary and secondary care sources of ascertainment with adjustment for death and migration. Diabet Med 2000;17:141-5.

8 Reincke M, Nieke J, Krestin GP, et al. Preclinical Cushing's syndrome in "adrenal incidentalomas": comparison with adrenal Cushing's syndrome. J Clin Endocrinol Metab 1992;75:826-32.

9 Nieman LK, Cutler GB. The sensitivity of the urine free cortisol measurement as a screening test for Cushing's syndrome. 72nd annual meeting of the Endocrine Society, Atlanta, 1990 (abst 822).

10 Cronin C, Igoe D, Duffy MJ, et al. The overnight dexamethasone test is a worthwhile screening procedure. Clin Endocrinol (Oxf) 1990;33:27-33. 